A. YAMADA

KODAI MATH, J.

9 (1986), 401-405

\title{
BLOCH CONSTANT AND VARIATION OF BRANCH POINTS
}

\author{
BY AKIRA YAMADA
}

\section{Introduction.}

Let $F$ be the set of functions $f$ regular in the unit disc $\Delta$ normalized by the conditions $f(0)=0$ and $f^{\prime}(0)=1$. For $z \in \Delta$, let $B(f)$ denote the least upper bound of the radii of all unramified disc centered at $f(z)$ which is contained in the Riemann surface of $f$. The Bloch constant $B$ is defined by

$$
B=\inf _{f \in F} B(f) .
$$

Although, the precise value of $B$ is not known, we have the estimate [4] $B>\frac{\sqrt{3}}{4}$. In 1937 Ahlfors-Grunsky [1] obtained an upper bound $B \leqq B(g)=0.47 \cdots$ by constructing a function $g \in F$ called the Ahlfors-Grunsky function. Also, they conjectured that $B=B(g)$. The function $g: \Delta \rightarrow C$ is obtained as follows. Let $S$ be the interior of the N.E. (non-Euclidian) triangle in $\Delta$ with the angles $\frac{\pi}{6}, \frac{\pi}{6}$ and $\frac{\pi}{6}$ and the vertices at $\sigma, \omega \sigma$ and $\omega^{2} \sigma$ where $\sigma=(\sqrt{3}+1)^{-1 / 2}$ and $\boldsymbol{\omega}=e^{2 \pi i / 3}$. Let $T$ be the interior of the regular triangle with the vertices at $\tau$, $\omega \tau, \omega^{2} \tau$ where

$$
\tau=\sigma \cdot \Gamma\left(\frac{1}{3}\right) \Gamma\left(\frac{11}{12}\right) / \Gamma\left(\frac{1}{4}\right)=0.47 \cdots
$$

By Schwarz's reflection the analytic function mapping $S$ conformally onto $T$ with $g\left(\omega^{k} \sigma\right)=\omega^{k} \tau(k=0,1,2)$ is continued analytically to a function $g \in F$ defined on $\Delta$, which is the Ahlfors-Grunsky function. It is well known that $g$ is a normal branched covering of the complex plane $C$ which is simply branched at every point of the regular triangular lattice $\{(n+\omega m) \tau \mid n, m \in \boldsymbol{Z}\}$ but is not branched elsewhere.

A. W. Goodman [3] introduced a variation of branch points for an analytic function in $\Delta$. We denote by $f_{c, \lambda}$ Goodman's branch variation of $f$.

The aim of this paper is to prove

THEOREM. For every branch point $c \in \Delta$ and sufficiently small $\lambda \in C(\lambda \neq 0)$, the Ahlfors-Grunsky function $g$ satisfies

Received February 17, 1986 


$$
B(g)<B\left(g_{c, \lambda} / g_{c, \lambda}^{\prime}(0)\right) \text {. }
$$

Note that $g_{c, \lambda} / g_{c, \lambda}^{\prime}(0) \in F$. The above Theorem asserts that the Bloch radius $B(f)$ attains a local minimum at the Ahlfors-Grunsky function $g$ when we vary its branch points slightly. For related results the reader is refered to $H$. Yanagihara's recent paper [7].

\section{Proof of Theorem.}

Goodman's branch variation [3] is described as follows. Let $f(z): \Delta \rightarrow \boldsymbol{C}$ be regular in $\Delta$ with $f^{\prime}(0)>0$, and map $\Delta$ onto a Riemann surface, having a simple branch point at $f(c)$. For $\lambda \in C$ sufficiently small we form a new Riemann surface $R^{*}$, from $R$, by moving the branch point at $f(c)$ to $f(c)+\lambda$, while holding the boundary and all other branch points of $R$ fixed. Since $R^{*}$ is simply-connected, there is a unique function $f_{c, \lambda}$ mapping $\Delta$ conformally onto $R^{*}$ such that $f_{c, \lambda}^{\prime}(0)>0$. The function $f_{c, \lambda}$ is called a Goodman's branch variation of $f$.

LEMMA 1. For sufficiently small $\lambda \in C$, we have

$$
f_{c, \lambda}(z)=f(z)-\frac{1}{2} z f^{\prime}(z)\left[A \cdot \frac{c+z}{c-z}+\bar{A} \cdot \frac{1+\bar{c} z}{1-\bar{c} z}\right]+0\left(\lambda^{2}\right),
$$

where $A=\frac{\lambda}{c^{2} f^{\prime \prime}(c)}$ and the estimate is uniform for $z$ in compact subsets of $\Delta$.

Proof. See [3]. For another derivation of the above formula using q.c. variation, see [6]. q. e. d.

In particular, for Ahlfors-Grunsky function $g$, Lemma 1 shows

$$
g_{c, \lambda}^{\prime}(0)=1-R e \frac{\lambda}{c^{2} f^{\prime \prime}(c)}+0\left(\lambda^{2}\right) \text {. }
$$

Let $r(x, y, z)$ denote the (Euclidian) radius of the circle passing through the points $x, y$ and $z(\in C)$. Since, if $\gamma z=a z+b(a, b \in C)$,

$$
r(\gamma x, \gamma y, \gamma z)=|a| r(x, y, z),
$$

we have

$$
\frac{\partial r}{\partial x}(\gamma x, \gamma y, \gamma z)=\frac{|a|}{a} \frac{\partial r}{\partial x}(x, y, z) .
$$

On the other hand, an elementary calculation gives

$$
\frac{\partial r}{\partial x}\left(1, \omega, \omega^{2}\right)=\frac{1}{6} \text {. }
$$

Thus, if we move the branch point $g(c)$ to $g(c)+\lambda$, the maximal radius $\rho(\lambda)$ of 
all unramified disks in $R^{*}$ is given by

$$
\rho(\lambda)=\tau+\max _{k \in Z} 2 \operatorname{Re}\left[\lambda \cdot \frac{1}{6} e^{k \pi \tau / 3}\right]+0\left(\lambda^{2}\right) .
$$

This follows from the fact that the branch points of $g$ forms a regular triangular lattice and from the identities (1.2) and (1.3).

LEMMA 2. If the inequality

$$
\left|c^{2} g^{\prime \prime}(c)\right|>2 \sqrt{3} \tau
$$

holds, then we have

$$
B(g)<B\left(g_{c, \lambda} / g_{c, \lambda}^{\prime}(0)\right)
$$

for sufficiently small $\lambda \in \boldsymbol{C}(\lambda \neq 0)$.

Proof. Since $B\left(g_{c, \lambda}\right)=\rho(\lambda)$ and $B(g)=\tau$, the identities (1.1) and (1.4) give

$$
\begin{aligned}
B\left(g_{c, \lambda} / g_{c, \lambda}^{\prime}(0)\right) & =B\left(g_{c, \lambda}\right) / g_{c, \lambda}^{\prime}(0) \\
& =\tau+\max _{k \in Z} \operatorname{Re} \frac{\lambda}{3}\left[\frac{3 \tau}{c^{2} g^{\prime \prime}(c)}+e^{k \pi i / 3}\right]+0\left(\lambda^{2}\right) \\
& \geqq B(g)+\frac{|\lambda|}{3}\left[\max _{k \in Z} \operatorname{Re} e^{(k \pi / 3)+\theta}-\left|\frac{3 \tau}{c^{2} g^{\prime \prime}(c)}\right|\right]+0\left(\lambda^{2}\right)
\end{aligned}
$$

with $\theta=\arg \lambda$. Since

$$
\max _{k \in Z} R e e^{(k \pi \imath / 3)+\theta} \geqq \frac{\sqrt{3}}{2} \quad \text { for any } \quad \theta \in \boldsymbol{R},
$$

(1.5) easily implies the desired inequality. q.e.d.

Let $\Gamma$ be the group of $N$. E. isometries of $\Delta$ generated by $l_{1}, l_{2}$ and $l_{3}$ where $l_{\imath}(i=1,2,3)$ are the reflections in each side of $N$. E. triangle $S$. We denote by $\Gamma_{0}$ the conformal subgroup of $\Gamma$. Poincaré's polygon theorem [5] implies that the group $\Gamma$ is discontinuous and that the triangle $S$ is a fundamental polygon for $\Gamma$.

LEMMA 3. For any $c \in \Delta$ with $g^{\prime}(c)=0$, we have

$$
\left|c^{2} g^{\prime \prime}(c)\right| \geqq\left|\sigma^{2} g^{\prime \prime}(\sigma)\right| \text {. }
$$

Proof. Observing that $c$ is of the form $\gamma\left(\omega^{k} \sigma\right)$ for some $\gamma \in \Gamma_{0}$ and $k \in \boldsymbol{Z}$, we may assume by symmetry that $c=\gamma \sigma\left(\gamma \in \Gamma_{0}\right)$. Next, note that, for $\gamma \in \Gamma_{0}$,

$$
g(\gamma z)=\omega^{k} g(z)+\text { const. }, \quad k \in \boldsymbol{Z} \text {. }
$$

Differentiating the above identity, we have

$$
g^{\prime \prime}(\gamma z)\left(\gamma^{\prime} z\right)^{2}+g^{\prime}(\gamma z) \gamma^{\prime \prime} z=\omega^{k} g^{\prime \prime}(z),
$$


so that, by $g^{\prime}(c)=0$,

$$
g^{\prime \prime}(c)\left(\gamma^{\prime} \sigma\right)^{2}=\omega^{k} g^{\prime \prime}(\sigma)
$$

Hence,

$$
\left|c^{2} g^{\prime \prime}(c)\right|=\frac{|c|^{2}}{\left(1-|c|^{2}\right)^{2}} \cdot\left(1-\sigma^{2}\right)^{2}\left|g^{\prime \prime}(\sigma)\right| .
$$

The Lemma will be proved if we show that $|c| \geqq \sigma$. This is easy. Let $z=\gamma_{0} \sigma$ $\left(\gamma_{0} \in \Gamma\right)$ be a point in $\Delta$ such that

$$
|z|=\min _{\gamma \in \Gamma}|\gamma \sigma| .
$$

The existence of such a point $z$ is clear from the discontinuity of the group $\Gamma$. Then we have

$$
d(z, 0) \leqq d\left(l_{i} \gamma_{0} \sigma, 0\right), \quad i=1,2,3,
$$

where $d(\cdot, \cdot)$ denotes the hyperbolic distance. Hence,

$$
d(z, 0) \leqq d\left(z, l_{i}(0)\right), \quad \imath=1,2,3,
$$

so that $z \in \bar{S}$. Since $S$ is a fundamental polygon, we conclude that $z=\sigma$, as desired. q.e.d.

LEMMA 4.

$$
g^{\prime \prime}(\sigma)=-(\sqrt{3}+1)^{2} \cdot 2^{-37 / 12} \cdot 3^{-5 / 8} \cdot \pi^{-5 / 2} \cdot \Gamma^{2}\left(\frac{1}{4}\right) \Gamma^{3}\left(\frac{1}{3}\right) .
$$

Proof. Here, our basic reference is [2]. Let $S_{1}$ be the interior of the N.E. triangle in $\Delta$ with the angles $\frac{\pi}{6}, \frac{\pi}{6}$ and $\frac{\pi}{6}$ that has its vertices at $0, \sigma_{1}, e^{\pi \imath / 6} \sigma_{1}$ with some positive constant $\sigma_{1}$, and let $T_{1}$ be the interior of the regular triangle with the vertices at $0, \tau, e^{\pi i / 3} \tau$. We denote by $g_{1}$ the function mapping $S_{1}$ conformally onto $T_{1}$ with $g_{1}(0)=0, g_{1}\left(\sigma_{1}\right)=\tau$ and $g_{1}\left(e^{\pi i / 6} \sigma_{1}\right)=e^{\pi i / 3} \tau$. Then it is easy to see that there exists a Möbius transformation $\varphi$ with $\varphi(0)=\sigma$ satisfying the identity

$$
g_{1}(z)=e^{i \theta}(g \circ \varphi(z)-\tau)
$$

with some real constant $\theta$. Differentiation shows

$$
g^{\prime \prime}(\boldsymbol{\sigma})=-\left(1-\sigma^{2}\right)^{-2} g_{1}^{\prime \prime}(0),
$$

since geometrically it is clear that $g^{\prime \prime}(\sigma)<0$ and $g_{1}^{\prime \prime}(0)>0$. On the other hand, we can express $g_{1}$ as a composition $v \circ u^{-1}$ where $u$ (resp. $v$ ) is the function mapping the upper half-plane $\{\operatorname{Im} z>0\}$ onto $S_{1}$ (resp. $T_{1}$ ) in such a way that the origin is kept fixed and the other vertices of the triangle correspond to 1 and $\infty$. By [2, Vol. II, pp. 162-163], $u(z)$ and $v(z)$ have the expansion at the origin, 
and

$$
u(z)=C_{1} z^{1 / 6}+\text { higher order terms, }
$$

$$
v(z)=C_{2} z^{1 / 3}+\text { higher order terms, }
$$

where the coefficients $C_{1}$ and $C_{2}$ are given explicitly by

$$
C_{1}=\frac{\sqrt{2}}{\sqrt{\sqrt{3}+1}} \cdot \frac{\Gamma(5 / 6) \Gamma(3 / 4)}{\Gamma(5 / 12) \Gamma(7 / 6)}
$$

and

$$
C_{2}=\frac{\sqrt{3}}{\sqrt{\sqrt{3}+1}} \cdot \frac{\Gamma(2 / 3) \Gamma(11 / 12)}{\Gamma(1 / 4) \Gamma(4 / 3)}
$$

Hence,

$$
g_{1}(z)=v \cdot u^{-1}(z)=C_{2} C_{1}^{-2} \cdot z^{2}+\text { higher order terms, }
$$

so that

$$
g_{1}^{\prime \prime}(0)=2 C_{2} C_{1}^{-2} .
$$

By applying standard formulas for the function $\Gamma(z)$, the identities (1.7)-(1.10) yield the value of $g^{\prime \prime}(\sigma)$, as desired. q.e.d.

Lemmas 2 and 3 imply that to conclude our THEOREM it is only necessary to show

$$
\left|\sigma^{2} g^{\prime \prime}(\sigma)\right|>2 \sqrt{3} \tau
$$

However, a computation using Lemma 4 gives

$$
\left|\sigma^{2} g^{\prime \prime}(\sigma)\right|=2.34 \cdots>2 \sqrt{3} \tau=1.63 \cdots .
$$

This completes the proof of the THEOREM.

\section{REFERENCES}

[1] L.V. Ahlfors and H. Grunsky, Über die Blochschen Konstante, Math. Z., 42 (1937), 671-673.

[2] C. CarathÉdory, Theory of functions, I, II, Chelsea, New York, 1954.

[3] A. W. Goodman, Variation of the branch points for an analytic function, Trans. Amer. Math. Soc., 89 (1958), 277-284.

[4] M. Heins, On a class of conformal metrics, Nagoya Math. J., 21 (1962), 1-60.

[5] B. MAsKiT, On Poincaré's theorem for fundamental polygons, Adv. in Math., 7 (1971), 219-230.

[6] A. YAMADA, Q.c. variations for analytic functions, to appear.

[7] H. YANAGIHARA, Local minimality of Ahlfors-Grunsky functions for affine variations, to appear. 